\author{
1 Department of Cardi- \\ ology, Heart Center \\ North Rhine-Westpha- \\ lia, Ruhr University of \\ Bochum, Bad Oeyn- \\ hausen, Germany, \\ ${ }^{2}$ Marienhospital Herne, \\ Ruhr University of Bo- \\ chum, Herne, Germany, \\ ${ }^{3}$ Department of Internal \\ Medicine,Kreiskranken- \\ haus Rinteln, Germany.
}

\section{Detection of a Type 1 Brugada ECG by ECG Recording at a Higher Intercostal Space of Leads $V_{1}$ and $V_{2}$}

\author{
Thomas Butz ${ }^{1,2}$, Jürgen Vogt ${ }^{1}$, Christian Vielhauer ${ }^{3}$, Ulrike Wetzel' ${ }^{1}$, Christoph Langer', \\ Dieter Horstkotte ${ }^{1}$
}



Figures 1a to 1d. a) ECG recorded at the 4th intercostal space and standard space, respectively, of $V_{1}$ and $V_{2}$. b) ECG recorded at the 3rd intercostal space (3. ICR) of $V_{1}$ and $\left.V_{2} . c\right)$ ECG recorded at the 2nd intercostal space (2. ICR) of $V_{1}$ and $V_{2}$ demonstrating the typical findings of a type 1 Brugada ECG. d) ECG recorded at the 4th intercostal space and standard space, respectively, showing a type 1 Brugada ECC during the ajmaline test ( $1 \mathrm{mg} / \mathrm{kg}$ i.v.; courtesy of Dr. C. Vielhauer, Kreiskrankenhaus Rinteln, Germany).

Herz 2010;35:112 DOI 10.1007/ s00059-010-3315-5

Address for Correspondence

Thomas Butz, MD Department of Cardiology and Angiology Marienhospital Herne Ruhr University of Bochum

Hoelkeskampring 40 44625 Herne, Germany Phone (+49/2323) 499-0,

Fax -360

E-mail:Thomas.Butz@ Marienhospital-Herne.de
The type 1 Brugada ECG pattern is characterized by the documentation of a spontaneous electrocardiogram (ECG) with specific ST segment elevation in the right precordial leads $\left(\mathrm{V}_{1}-\mathrm{V}_{3}\right)$ and a J-point elevation of $0.2 \mathrm{mV}$ in the absence or presence of sodium channel blockers ("coved type") $[1,2]$. Brugada syndrome is defined as a typical ECG finding, the exclusion of a structural heart disease, and syncope or sudden cardiac death.

We present the ECG findings of a 52-year-old man who was admitted to our center after a first episode of syncope. Invasive diagnostics ruled out a coronary heart disease, left ventricular function was normal. ECG recordings at the 4th intercostal space, which is the standard lead position (Figure 1a), and at the 3 rd intercostal space (Figure $1 \mathrm{~b}$ ) of $\mathrm{V}_{1}$ and $\mathrm{V}_{2}$ were not path-breaking. By contrast, ECG recordings at a higher space (2nd intercostal space, Figure 1c) of leads $\mathrm{V}_{1}$ and $\mathrm{V}_{2}$ demonstrated a type 1 Brugada ECG.

A positive ajmaline test (ajmaline $1 \mathrm{mg} / \mathrm{kg}$ i.v.) showing a type 1 Brugada ECG with specific ST segment elevation in the right precordial leads $\left(\mathrm{V}_{1}-\mathrm{V}_{3}\right)$ with a coved type and a J-point elevation of $0.2 \mathrm{mV}$ had previously been performed in the admitting hospital (Figure 1d) [1, 2]. After the diagnosis of a Brugada syndrome, a cardioverter defibrillator was implanted to prevent sudden cardiac death.

Recently, it has been shown that an ECG recording at a higher intercostal space in leads $\mathrm{V}_{1}$ and $\mathrm{V}_{2}$ had higher sensitivity than that at a standard space in these leads in unmasking and detecting a type 1 Brugada ECG. Moreover, a type 1 Brugada ECG recorded only at a higher space in leads $V_{1}$ and $V_{2}$ showed a similar prognostic value for subsequent cardiac events as that recorded at a standard space in these leads [3, 4]. Higher recordings of leads $V_{1}$ and $V_{2}$ are recommended as an alternative to drug challenge testing with sodium channel blockers, which should only be considered as next diagnostic test, if the result of higher ECG recordings is negative.

Because of the potentially life-threatening risk of the ajmaline test, it should only be performed, if an ECG at a higher intercostal space has already been recorded [4].

\section{References}

1. Brugada P, Brugada J. Right bundle branch block, persistent ST segment elevation and sudden cardiac death: a distinct clinical and electrocardiographic syndrome. A multicenter report. J Am Coll Cardiol 1992;20:1391-6.

2. Brugada R, Brugada J, Antzelevitch C, et al. Sodium channel blockers identify risk for sudden death in patients with ST-segment elevation and right bundle branch block but structurally normal hearts. Circulation 2000;101:510-5.

3. Miyamoto K, Yokokawa M, Tanaka K, et al. Diagnostic and prognostic value of a type 1 Brugada electrocardiogram at higher (third or second) $V_{1}$ to $V_{2}$ recording in men with Brugada syndrome. Am J Cardiol 2007;99:53-7.

4. Shimizu W, Matsuo K, Takagi M, et al. Body surface distribution and response to drugs of ST segment elevation in Brugada syndrome: clinical implication of eighty-seven-lead body surface potential mapping and its application to twelve-lead electrocardiograms. J Cardiovasc Electrophysiol 2000;11:396-404. 